\title{
The Brief Case: Laryngeal Blastomycosis Involving a Vocal Cord Implant
}

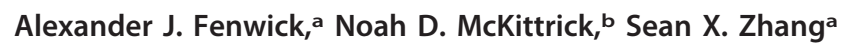 \\ aDepartment of Pathology, Division of Microbiology, The Johns Hopkins Hospital, Baltimore, Maryland, USA \\ bDepartment of Medicine, Division of Infectious Diseases, The Johns Hopkins Hospital, Baltimore, Maryland, USA
}

KEYWORDS Blastomyces, blastomycosis, larynx

\section{CASE}

54-year-old woman from southern Ohio presents with progressive dysphonia and a chronic nonhealing neck wound. She has a history of Hodgkin's lymphoma treated with external beam radiation of the neck 30 years ago. Beginning 3 to 4 years ago, she began to experience dysphonia and was diagnosed with left true vocal cord paralysis, thought to be a late complication of her radiation. She subsequently underwent left medial thyroplasty and placement of a laryngeal prosthetic, which improved her symptoms.

A few months before her current presentation, however, her dysphonia returned. Her surgeon suspected displacement of the laryngeal prosthesis as the cause, so the original implant was replaced. Approximately 5 weeks postoperatively, she developed induration, erythema, and tenderness of the anterior neck surgical incision. Empiric cephalexin provided initial improvement, but she soon developed wound dehiscence with purulent drainage. A course of amoxicillin-clavulanate offered no improvement. Over the following weeks, the wound alternated between actively draining and crusting over, resulting in a 2-cm ulcer.

The patient was referred to otolaryngology shortly after these episodes. Direct laryngoscopy at her initial visit was notable for a stiff and polypoid left true vocal fold, and a trial of clindamycin followed by oral prednisone failed to relieve her symptoms. Computed tomography imaging demonstrated mild biapical scarring of the lungs and the left vocal fold implant with overlying soft tissue inflammation extending to the skin; no fistulous tract was seen. She was admitted for debridement of the neck lesions and biopsy sampling of the vocal fold mass; grossly, the lesion was exophytic and friable, involving the anterior $50 \%$ of the true vocal cord extending from superior to medial. Biopsy specimens of the mass and the surgical wound site revealed ulcerated squamous mucosa with chronic granulomatous inflammation, reactive epithelial changes, and numerous round 5 - to $10-\mu \mathrm{m}$ refractile yeast forms with broad-based budding that were poorly stained by hematoxylin and eosin (H\&E) but were Gomori methenamine silver (GMS) positive, suggestive of Blastomyces yeast cells (Fig. 1A and B).

She underwent a second, more extensive debridement and removal of the new laryngeal implant, with the sebsequent operative report noting a fistulous tract extending from the chronic ulcer to an area of the left thyroid ala bordering the area of the implanted prosthetic. Tissue fungal cultures were positive for growth after 2 weeks of incubation, with cottony white colonies on both Sabouraud and brain heart infusion agar slants (Fig. 2A). Lactophenol cotton blue staining of a slide culture preparation demonstrated septate hyphae with smooth round conidia arising terminally on short lateral conidiophores in a characteristic "Iollipop-on-astick" arrangement (Fig. 2B), suggestive of Blastomyces dermatitidis/gilchristii. This was subsequently confirmed by a Blastomyces-specific DNA probe (AccuProbe, Gen-Probe, Inc., San Diego, CA).

Citation Fenwick AJ, McKittrick ND, Zhang SX. 2019. The Brief Case: Laryngeal blastomycosis involving a vocal cord implant. J Clin Microbiol 57:e00409-19. https://doi.org/10.1128/JCM .00409-19.

Editor Carey-Ann D. Burnham, Washington University School of Medicine Copyright $\odot 2019$ American Society for Microbiology. All Rights Reserved. Address correspondence to Alexander J. Fenwick, afenwic3@jhmi.edu.

For answers to the self-assessment questions and take-home points, see https://doi.org/10.1128/ JCM.00412-19 in this issue.

Published 23 October 2019 

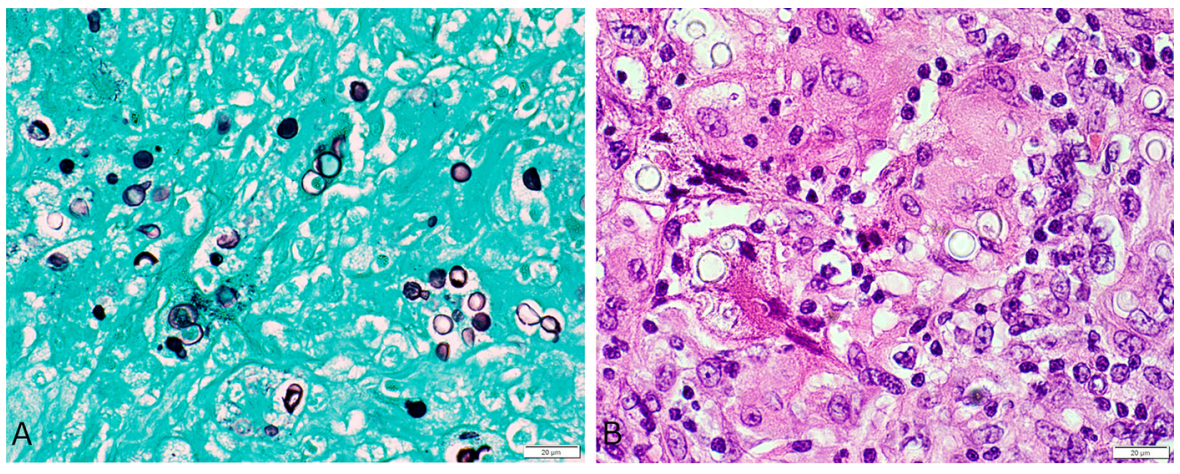

FIG 1 Biopsy of left neck incisional scar. (A) GMS stain, magnification $\times 600$. Numerous round GMS-positive forms $\sim 5$ to $15 \mu \mathrm{m}$ in diameter, consistent with yeast. (B) H\&E stain, magnification $\times 600$. Scar tissue with chronic granulomatous inflammation and giant cells; numerous round, poorly stained, and refractile forms ranging from 5 to $15 \mu \mathrm{m}$ in diameter, highly suggestive of yeast.

The patient was started on twice-daily oral itraconazole $200 \mathrm{mg}$ for presumptive laryngeal blastomycosis, but she developed severe peripheral edema as an adverse reaction to the itraconazole. The remainder of her treatment was completed with posaconazole $300 \mathrm{mg}$ daily. A new laryngeal implant was placed following therapy, and a skin biopsy specimen of the scar taken at the time was negative for yeast.

\section{DISCUSSION}

Blastomycosis is a systemic infection caused by the dimorphic fungus $B$. dermatitidis, which was first described by Gilchrist and Stokes (1). Additionally, a closely related and phenotypically identical species, B. gilchristii, was recently described (2), causing a similar clinical presentation (3). Blastomyces isolates are the etiological agents of one of the three major mycoses endemic in North America, with a geographic range centered around the Great Lakes and the Mississippi-Ohio River valleys, showing considerable overlap with that of Histoplasma species $(3,4)$. It is found in the decaying organic litter of wooded areas, especially near fresh water $(3,4)$. Blastomyces isolates are thermally dimorphic, growing as filamentous mold in the environment and as yeast in the body $\left(35^{\circ} \mathrm{C}\right.$ to $\left.37^{\circ} \mathrm{C}\right)$ of its mammalian host.

Exposure to and infection with Blastomyces isolates typically occur via inhalation of
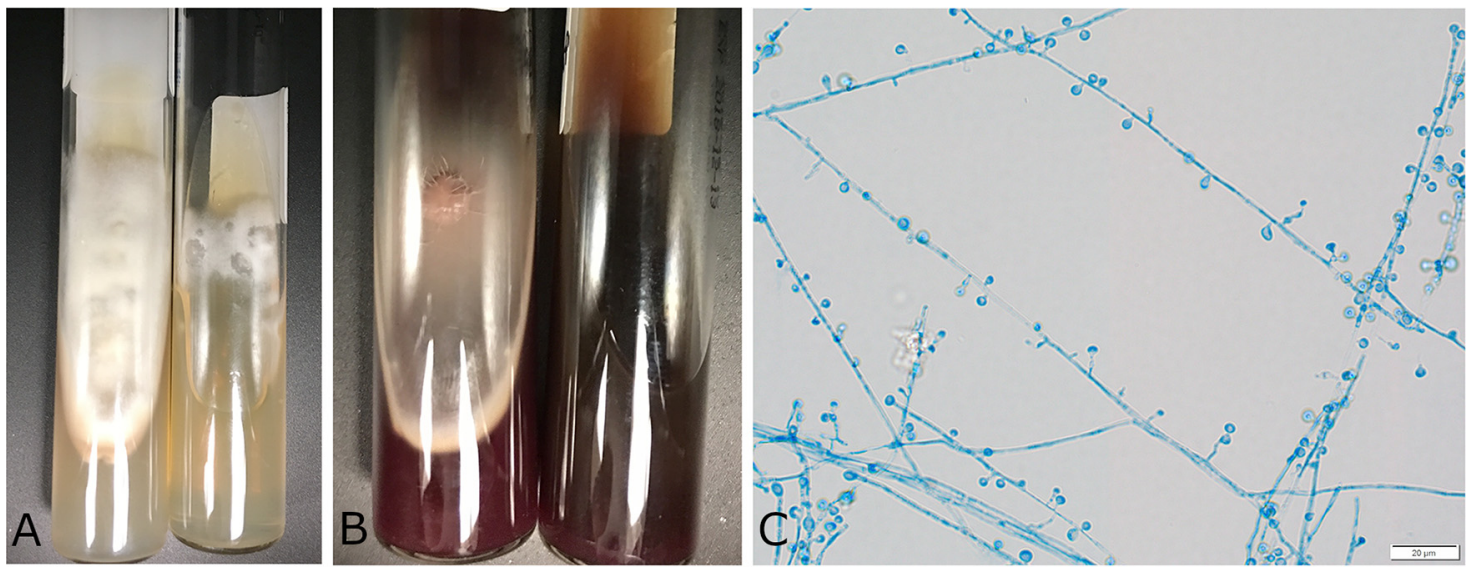

FIG 2 (A and B) Blastomyces dermatitidis/gilchristii mold-phase colony morphology at $30^{\circ} \mathrm{C}$ incubation. Colonies are first detected days to weeks after initial specimen inoculation. Blastomyces dermatitidis isolates are thermally dimorphic, with fluffy white colonies and fine tan-white aerial mycelium seen in the mold phase (pictured) and smooth-to-waxy creamy-tan colonies seen in the yeast phase after incubation at $35^{\circ} \mathrm{C}$ to $37^{\circ} \mathrm{C}$. (A) Inhibitory mold agar; (B) brain heart infusion agar. (C) B. dermatitidis/gilchristii's filamentous growth has septate hyaline mycelium ( $\sim 1$ to $2 \mu \mathrm{m}$ in diameter) that produces smooth and round/oval microconidia ( 2 to $4 \mu \mathrm{m}$ in diameter). The characteristic "lollipop-on-a-stick" appearance is a product of the terminal microconidia on lateral conidiophores (lactophenol cotton blue, magnification $\times 600$ ). 
aerosolized infectious conidia after disturbance of contaminated organic debris $(3,4)$. Traumatic inoculation is rare but has been reported (3). Conversion to the yeast phase enables the organism to evade the host immune response, proliferate, and upregulate various virulence factors (4), resulting in primary infection. The most important risk factor for acquiring Blastomyces infection is environmental exposure in an area of endemicity $(3,4)$. Other reported risk factors include immunocompromised status and collagen vascular disease, although most patients who develop symptomatic infection are immunocompetent (4). Blastomyces isolates are capable of infecting numerous mammals besides humans. In fact, blastomycosis of pet dogs has been identified as a predictor for human infection and is often informally considered a harbinger of disease (4). Interestingly, our patient reported having recently finished giving her dog a course of antifungal therapy for disseminated blastomycosis, which was acquired after hiking through nearby woods.

Primary blastomycosis typically involves the lungs and can cause a wide spectrum of nonspecific clinical manifestations, ranging from subclinical infection ( $~ 50 \%$ of patients) to severe respiratory distress, which is easily misdiagnosed (3). Patients generally present with a mild, febrile respiratory illness with evidence of consolidation seen on chest radiographs, mimicking community-acquired bacterial pneumonia. More severe disease may present similarly to tuberculosis or malignancy, with hemoptysis, anorexia, weight loss, and pulmonary nodules seen on imaging (3). Notably, our patient did not present with any obvious symptomatic or radiological evidence of pulmonary involvement.

Disseminated blastomycosis is the result of hematogenous spread and occurs in up to $40 \%$ of symptomatic infections (3). The most commonly involved extrapulmonary sites are the skin, bones, genitourinary tract, and central nervous system. Mucocutaneous disease is easily mistaken for carcinoma because the lesions tend to rapidly develop, appear as well-circumscribed verrucous or pustular lesions with irregular heaped-up borders and purple-red discoloration, are commonly ulcerated, and bleed easily (3-5).

To our knowledge, this is the first reported case of blastomycosis associated with a prosthetic laryngeal implant. Primary laryngeal blastomycosis, although relatively rare, is the most common site of head and neck involvement (5). Unlike most extrapulmonary manifestations of the disease, laryngeal blastomycosis is presumed to result from direct inoculation of the laryngeal mucosa via inhalation. It invariably presents as worsening dysphonia and an exophytic polypoid mass on either the true or false vocal cords (5). Biopsy specimens show pseudoepitheliomatous hyperplasia with atypia, which may be easily mistaken for malignancy, particularly if no special staining (i.e., GMS, periodic acid-Schiff stain [PAS]) is performed. Chronic infection, frequently the result of misdiagnosis, can lead to extension into the surrounding tissues, including fistulization to the overlying skin, as was seen in this patient (5). In the review from 2000, several patients had undergone radical neck dissections, chemotherapy, and/or radiation therapy for presumptive malignancy before the correct diagnosis was made (5). Although infection was at the top of the differential diagnosis in our patient, a biopsy specimen of the mass was sent for histopathological evaluation and culture so that malignancy could be ruled out.

Diagnosis of blastomycosis relies on the demonstration of organism through growth in culture, positive serological testing, and/or microscopic identification of yeast in tissue $(3,4)$. Blastomyces isolates grow moderately fast in culture, with waxy off-white mycelial colonies appearing after 7 to 14 days of incubation at $25^{\circ} \mathrm{C}$ to $30^{\circ} \mathrm{C}$, although it can take up to 30 days. As the colonies mature and form aerial hyphae, they become fluffy in appearance (4). $\mathrm{KOH}$ or lactophenol cotton blue staining reveals narrow (1- to $2-\mu \mathrm{m})$ septate hyphae and short lateral conidiophores bearing single-terminal conidia ( 2 to $4 \mu \mathrm{m}$ ), giving the characteristic "lollipop-on-a-stick" appearance (4). Conversion of mycelial colonies to the yeast phase via incubation at $37^{\circ} \mathrm{C}$ reveals creamy tan and wrinkled yeastlike colonies (4); however, this is not practical for clinical use, because it can take weeks to months. Furthermore, excessive handling of the filamentous form is ill advised given its highly infectious nature. Confirmation from mycelial culture can be 
performed using fluorescent DNA probes or via internal-transcribed-spacer region sequencing; however, they cannot reliably distinguish $B$. dermatitidis from B. gilchristii isolates. Species differentiation is only achieved using whole-genome sequencing (2), although it has limited clinical relevance.

Serological antibody testing for Blastomyces isolates generally suffers from poor sensitivity, poor specificity, or both. Complement fixation (CF) is neither sensitive nor specific; newer immunodiffusion (ID) assays have improved specificity; however, they, too, suffer from poor sensitivity, and neither CF nor ID assays have much diagnostic utility, especially in regions of endemicity, which have high rates of seropositivity $(3,4)$. A recently developed enzyme immunoassay detecting an anti-BAD- 1 antibody has shown improved sensitivity and specificity, although it is not yet commercially available in the United States (3). Antigen testing for serum or urine shows increased sensitivity and specificity versus antibody testing; however, it suffers from a high degree of cross-reactivity, particularly with other dimorphic fungi (3). Additionally, blastomycosis has been noted as a significant cause of false-positive Histoplasma antigen tests (4). Other fungal biomarkers, such as $\beta$-D-glucan (BDG), have limited utility because Blastomyces yeast lacks BDG as a major cell wall component (4).

Histological or cytological examination of affected tissues often shows a granulomatous inflammation. Yeast forms, 5 to $15 \mu \mathrm{m}$ in diameter, with characteristic "broadbased budding" can be seen on GMS-stained sections. Staining with H\&E, PAS, and Papanicolaou stains highlights the thick refractile capsule surrounding the yeast (4).

The Infectious Diseases Society of America (IDSA) treatment guidelines recommend against necessarily treating mild or self-limited primary infection in immunocompetent hosts (6). For more mild to moderate disease requiring therapy, 6 to 12 months of oral itraconazole ( $200 \mathrm{mg}$ twice daily) is the treatment of choice. Treatment of moderate to severe infection requires 1 to 2 weeks of intravenous lipid amphotericin B (3 to $5 \mathrm{mg} / \mathrm{kg}$ ) followed by 6 to 12 months of twice-daily oral itraconazole (200 mg) (6). Other azoles, such as voriconazole, isavuconazole, and posaconazole, have in vitro activity against Blastomyces infection, but clinical data to support their use are limited, so treatment with these agents is not recommended.

\section{SELF-ASSESSMENT QUESTIONS}

1. Blastomyces dermatitidis/gilchristii has a geographic area of endemicity that largely overlaps that of which other thermally dimorphic fungus?
a. Coccidioides immitis/posadasii
b. Histoplasma capsulatum
c. Talaromyces (Penicillium) marneffei
d. Paracoccidioides brasiliensis
e. Cryptococcus neoformans

2. Diagnostic testing for Blastomyces isolates in serum generally suffers from moderately poor specificity. Additionally, infection with Blastomyces dermatitidis/ gilchristii often causes false-positive serological test results for which other pathogenic microorganism?
a. Aspergillus spp.
b. Cryptococcus neoformans
c. Histoplasma capsulatum
d. Legionella spp.
e. Pneumocystis jirovecii

3. What is the 2008 IDSA guideline-recommended therapy and duration for mild to moderate blastomycosis?

a. Lipid or deoxycholate amphotericin B (3 to $5 \mathrm{mg} / \mathrm{kg}, 1$ to 2 weeks), followed by itraconazole ( $200 \mathrm{mg}, 12$ months)

b. Lipid amphotericin B (3 to $5 \mathrm{mg} / \mathrm{kg}$ daily) 
c. Liposomal amphotericin B ( 3 to $5 \mathrm{mg} / \mathrm{kg}, 4$ to 6 weeks), followed by an oral

azole (200 $\mathrm{mg}$, at least 1 year)

d. Itraconazole (200 mg, 6 to 12 months)

e. Micafungin ( $400 \mathrm{mg}, 3$ to 4 months)

\section{REFERENCES}

1. Gilchrist TC, Stokes WR. 1898. A case of pseudo-lupus vulgaris caused by a Blastomyces. J Exp Med 3:53-78. https://doi.org/10.1084/jem.3.1.53.

2. Brown EM, McTaggart LR, Zhang SX, Low DE, Stevens DA, Richardson SE. 2013. Phylogenic analysis reveals a cryptic species Blastomyces gilchristii, sp. nov. within the human pathogenic fungus Blastomyces dermatitidis. PLoS One 8:e59237. https://doi.org/10.1371/journal.pone.0059237.

3. McBride JA, Gauthier GM, Klein BS. 2017. Clinical manifestations and treatment of blastomycosis. Clin Chest Med 38:435-449. https://doi.org/ 10.1016/j.ccm.2017.04.006.

4. Saccente M, Woods GL. 2010. Clinical and laboratory update on blasto- mycosis. Clin Microbiol Rev 23:367-381. https://doi.org/10.1128/CMR .00056-09.

5. Rucci J, Eisinger G, Miranda-Gomez G, Nguyen J. 2014. Blastomycosis of the head and neck. Am J Otolaryngol 35:390-395. https://doi.org/10 .1016/j.amjoto.2013.12.013.

6. Chapman SW, Dismukes WE, Proia LA, Bradsher RW, Pappas PG, Threlkeld MG, Kauffman CA. 2008. Clinical practice guidelines for the management of blastomycosis: 2008 update by the Infectious Disease Society of America. Clin Infect Dis 46:1801-1812. https://doi.org/10 $.1086 / 588300$. 